\title{
PDE APPROXIMATION OF LARGE SYSTEMS OF DIFFERENTIAL EQUATIONS
}

\author{
András BÁtKai, Ágnes Havasi, Róbert Horváth, DÁvid \\ Kunszenti-KovÁcs AND PÉTER L. Simon
}

\begin{abstract}
A large system of ordinary differential equations is approximated by a parabolic partial differential equation with dynamic boundary condition and a different one with Robin boundary condition. Using the theory of differential operators with Wentzell boundary conditions and similar theories, we give estimates on the order of approximation. The theory is demonstrated on a voter model where the Fourier method applied to the PDE is of great advantage.
\end{abstract}

Mathematics subject classification (2010): 47D06, 47N40, 65J10. ferences.

Keywords and phrases: Dynamics on networks, $C_{0}$-semigroups, approximation theorems, finite dif-

\section{REFERENCES}

[1] W. Arendt, G. Metafune, D. Pallara, S. Romanelli, The Laplacian with Wentzell-Robin boundary conditions on spaces of continuous functions, Semigroup Forum 67 (2003), 247-261.

[2] W. ARENDT, M. WARMA, The Laplacian with Robin boundary conditions on arbitrary domains, Potential Analysis 19 (2003), 341-363.

[3] A. BÁtKAI, P. CsOmós, B. FARKAS, G. NicKel, Operator splitting for nonautonomous evolution equations, J. Funct. Anal. 260 (2011), 2163-2190.

[4] A. Bátkai, P. Csomós, B. Farkas, A. Ostermann, Operator Semigroups for Numerical Analysis, Internet-Seminar Manuscript, 2012, https://isem-mathematik.uibk.ac .at.

[5] A. BÁtKAI, P. Csomós, G. NiCKel, Operator splittings and spatial approximations for evolution equations, J. Evol. Equ. 9, 3 (2009), 613-636.

[6] A. BÁtKAI, K.-J. ENGEL, Abstract wave equations with generalized Wentzell boundary conditions, J. Diff. Eqs. 207 (2004), 1-20.

[7] A. BÁtKAi, I. Z. Kiss, E. Sikolya, P. L. Simon, Differential equation approximations of stochastic network processes: an operator semigroup approach, Networks and Heterogeneous Media (NHM) 7 (2012), 43-58. doi: 10.3934/nhm.2012.7.43.

[8] R. Courant, K. Friedrichs, H. LEWY, Über die partiellen Differenzengleichungen der mathematischen Physik, Math. Ann. 100 (1928), 32-74.

[9] K.-J. EnGEL, Second order differential operators on C[0,1] with Wentzell-Robin boundary conditions, Evolution Equations: Proceedings in Honor of J. A. Goldstein's 60th Birthday (G. Ruiz Goldstein, R. Nagel, and S. Romanelli, eds.), Lect. Notes in Pure and Appl. Math., no. 234, Marcel Dekker, 2003, pp. 159-165.

[10] K.-J. ENGEL, The Laplacian on $C(\bar{\Omega})$ with generalized Wentzell boundary conditions, Arch. Math. 81 (2003), 548-558.

[11] K.-J. ENGEL, R. NAGEL, One-Parameter Semigroups for Linear Evolution Equations, Graduate Texts in Math., vol. 194, Springer-Verlag, 2000.

[12] R. Holley, T. Liggett, Ergodic theorems for weakly interacting infinite systems and the voter model, Ann. Probab. 3 (1975), 643-663.

[13] P. MandL, Analytical Treatment of One-Dimensional Markov-Processes, Springer Verlag, 1968.

[14] N. NAGY, I. Z. Kiss, P. L. SimON, Approximate master equations for dynamical processes on graphs, Math. Model. Nat. Phenom. 9 (2014), 32-46. 
[15] F. VAZQueZ, V. M. EguiluZ, Analytical solution of the voter model on uncorrelated networks, New J. Phys. 10 (2008), 063011.

[16] M. WARMA, Wentzell-Robin boundary conditions on C $[0,1]$, Semigroup Forum 66 (2002), 162-170. 